\title{
Exploración geológica en los stockpiles de la Mina Rosita, Nicaragua
}

\author{
Geological exploration in the stockpiles of the Rosita Mine, Nicaragua
}

\author{
Ezequiel Ernesto Alvarado Martínez¹, Jaime César Mayorga Rojas²
}

Recibido: 22/10/2021 - Aprobado: 19/07/2021 - Publicado: 23/12/2021

\begin{abstract}
RESUMEN
Los proyectos mineros requieren de grandes inversiones y su desarrollo o cierre están en dependencia entre otros factores a la variación en el precio de los commodities en el mercado internacional y al agotamiento de sus reservas mineras respectivamente. La Mina Rosita ubicada en el noreste de Nicaragua es buen ejemplo de cómo el precio de los metales y otros factores de tipo social entre otros, pueden afectar el desarrollo de una mina, ocasionando hasta su cierre definitivo, la Mina Rosita inició operaciones industriales a finales de la década de 1950 y cerro a mediados de la década de 1970. Las condiciones geológicas y tectónicas de Nicaragua han permitido la evolución y emplazamiento de diferentes tipos de yacimientos metálicos, caracterizando al país como el mayor productor de oro a nivel centroamericano durante el siglo pasado. La Mina Rosita está caracterizada por un yacimiento tipo Skarn Cu-Au-Ag emplazado en rocas de la formación Todos Santos del Cretáceo, así como en rocas volcánicas que han sido cortadas por intrusivos de composición intermedia y félsica de edad más reciente. Al momento del cierre de la Mina Rosita, en los alrededores del tajo Santa Rita se abandonaron volúmenes considerables de mineral de baja ley, así como varios waste dumps ${ }^{3}$ (vertederos) que con los nuevos métodos de exploración y avances tecnológicos ha sido posible determinar su volumen y ley para definirlos como recursos mineros que pueden ser aprovechados industrialmente.
\end{abstract}

Palabras claves: Mina; precios; recursos; reservas; vertederos; yacimientos.

\begin{abstract}
Mining projects require high investments, and their development or closure is dependent, among other factors, due to the variation in the price of commodities in the international market and due to the depletion of their mining reserves, respectively. The Rosita Mine located in the northeast of Nicaragua is a good example of how the price of metals and other social factors, among others, can affect the development of a mine, and may lead to its final closure, the Rosita Mine began industrial operations at late 1950s and closed in the mid-1970s. The geological and tectonic conditions of Nicaragua have allowed the evolution and location of different types of metallic deposits, characterizing the country as the largest gold producer in Central America during the last century. The Rosita Mine is characterized by a Skarn Cu-Au-Ag deposit located in rocks of the Todos Santos Cretaceous formation, as well as in volcanic rocks that have been cut by intrusives of intermediate and felsic composition of more recent age. At the time of closure of the Rosita Mine, in the surroundings of the Santa Rita pit, considerable volumes of low-grade ore were abandoned, as well as several waste dumps (landfills) that with new exploration methods and technological advances it has been possible to determine their volume and law to define them as mineral resources that can be exploited industrially.
\end{abstract}

Keywords: Commodities; deposits; mine; reserves; resources; waste dumps.

1 Universidad Nacional Mayor de San Marcos, Facultad de Ingeniería Geológica, Minera, Metalúrgica y Geográfica, Unidad de Posgrado, Lima, Perú. Alumno. Autor para correspondencia: eeam86@gmail.com

2 Universidad Nacional Mayor de San Marcos, Facultad de Ingeniería Geológica, Minera, Metalúrgica y Geográfica, EAPIG, Lima, Perú. Docente.

E-mail: jaime.mayorga@unmsm.edu.pe - ORCID: https://orcid.org/0000-0001-8423-3343

3 Mining waste dumps por su nombre en inglés o vertederos por su nombre en español. 


\section{INTRODUCCIÓN}

Las circunstancias políticas y sociales ocurridas en Nicaragua a finales de la década de los 70s llevaron consigo un cambio de gobierno mediante una revolución armada, que trajo como consecuencia la afectación del sector minero, ya que el nuevo gobierno nacionalizó las minas y las compañías se vieron obligadas a abandonar el país. El nuevo gobierno, aunque realizó algunos esfuerzos por continuar operando las minas no contaba con recursos económicos ni capacidad técnica suficiente para continuar su operación y adicionalmente el país continuaba inmerso en una guerra civil que se libró principalmente en las montañas de Nicaragua.

Producto de estos problemas la Mina Rosita cerro definitivamente sus operaciones en el año 1981, las instalaciones de la planta de procesamiento fueron parcialmente trasladadas a otras minas o fueron destruidas o vendidas como chatarra. De igual manera, todas las obras mineras quedaron abandonadas, como el tajo Santa Rita (nombrado de esa manera por el cerro homónimo) que al no estar en operación y no continuar bombeando el agua se inundó de manera natural, dando origen a un embalse, alrededor del tajo Santa Rita quedaron abandonados volúmenes considerables de mineral de baja ley, waste dumps y colas ${ }^{4}$.

Las dimensiones aproximadas del tajo Santa Rita son de $250 \mathrm{~m}$ de ancho y $370 \mathrm{~m}$ de longitud y una profundidad de $120 \mathrm{~m}$.

La Mina Rosita operó entre 1959 y 1975, el mineral extraído y no procesado (mena y ganga) por considerarse de baja ley fue depositado en los alrededores del tajo Santa Rita y sus colas depositadas al extremo este de la planta de tratamiento.

Es importante mencionar que el precio del cobre en el mercado mundial fue otro factor que afectó la operación minera, trayendo como consecuencia mayor volumen de mineral de baja ley que no fue procesado, en este artículo esos volúmenes de mineral quebrado y no procesado son nombrados por su nombre en inglés como stockpiles (reservas).

Como en toda actividad comercial, el costo beneficio es un factor para la toma de decisiones, en la actualidad el precio de los metales juega un papel determinante, por lo que se consideró a partir del año 2011 realizar nuevos trabajos de exploración para determinar el potencial de los antiguos stockpiles de la Mina Rosita, de manera general se desarrollaron 3 etapas de perforación (2011, 2012 y 2015) en los stockpiles y dos de muestreo en los antiguos tailings (relaves) (2012 y 2013).

La primera y segunda campaña de perforación en los stockpiles (2011 y 2012) tuvo como finalidad delimitar, cuantificar y definir el potencial de los stockpiles, los trabajos de perforación sirvieron de base para una primera cuantificación de recursos.

4 No se aborda en este artículo los pasivos ambientales dado que no es el tema objeto de estudio.
También durante este periodo se realizó un primer muestreo de los tailings para delimitar el área con mayor potencial, aunque no se delimitaron recursos mineros.

La tercera campaña de perforación (2015) permitió elevar la categoría de los recursos en los stockpiles de inferidos a indicados y en el caso de los tailings se densificó la red de muestreo y se calcularon recursos inferidos en una pequeña zona.

Los análisis y resultados obtenidos en la tercera etapa de exploración geológica demostraron que los recursos existentes (stockpiles y tailing), presentan una viabilidad económica, debido a que su ley mínima, factores modificadores y procesos metalúrgicos permiten continuar con una fase de prefactibilidad, factibilidad y finalmente de extracción y procesamiento minero.

\section{METODOS}

\subsection{Exploración geológica detallada en los stockpiles de Santa Rita}

La primera y segunda etapa (2011-2012) de los trabajos de exploración en los stockpiles contempló los siguientes trabajos:

1. Levantamiento topográfico, mediante este trabajo se determinó el área y volumen aproximado de cada stockpile. El nombre de los stockpiles está dado según su ubicación con respecto al tajo de Santa Rita, siendo estos: stockpile norte, este, sur, suroeste, noreste y norte No.2. Ver Figura 1

2. Muestreo superficial mediante canales continuos, tomando muestras a $1 \mathrm{~m}$ en la vertical y 0.15 $\mathrm{m}$ de profundidad, se realizaron un total de 17 canales que brindaron una idea general de la mineralización en los stockpiles muestreados. Ver Figura 2.

3. Con la delimitación morfo métrica de los stockpiles se planificó el primer programa de perforación de tipo Circulación Inversa (RC), con una grid de separación de aproximadamente 100 $\mathrm{m}$. Todos los pozos se perforaron con un ángulo de $90^{\circ}$ y la profundidad varió entre $10 \mathrm{~m}$ y $3.0 \mathrm{~m}$, los pozos terminaron en la roca madre y por ende atravesaron todo el stockpile. Ver figura No.3

4. Toma de muestras para cálculo de densidad, obteniendo una densidad promedio de $2.27 \mathrm{~g} / \mathrm{cm}^{3}$.

5. Se realizó un primer modelamiento de los stockpiles y una primera evaluación de recursos. (2015) contempló las siguientes actividades:

6. Densificación de la red de perforación RC (perforación infield), con una red de perforación de aproximadamente $50 \mathrm{~m}$ entre pozo. Al igual que la primera campaña el objetivo era atravesar todo el stockpile y terminar el pozo en la roca madre. La profundidad de los pozos varió entre $12.0 \mathrm{~m}$ y $34.5 \mathrm{~m}$. 


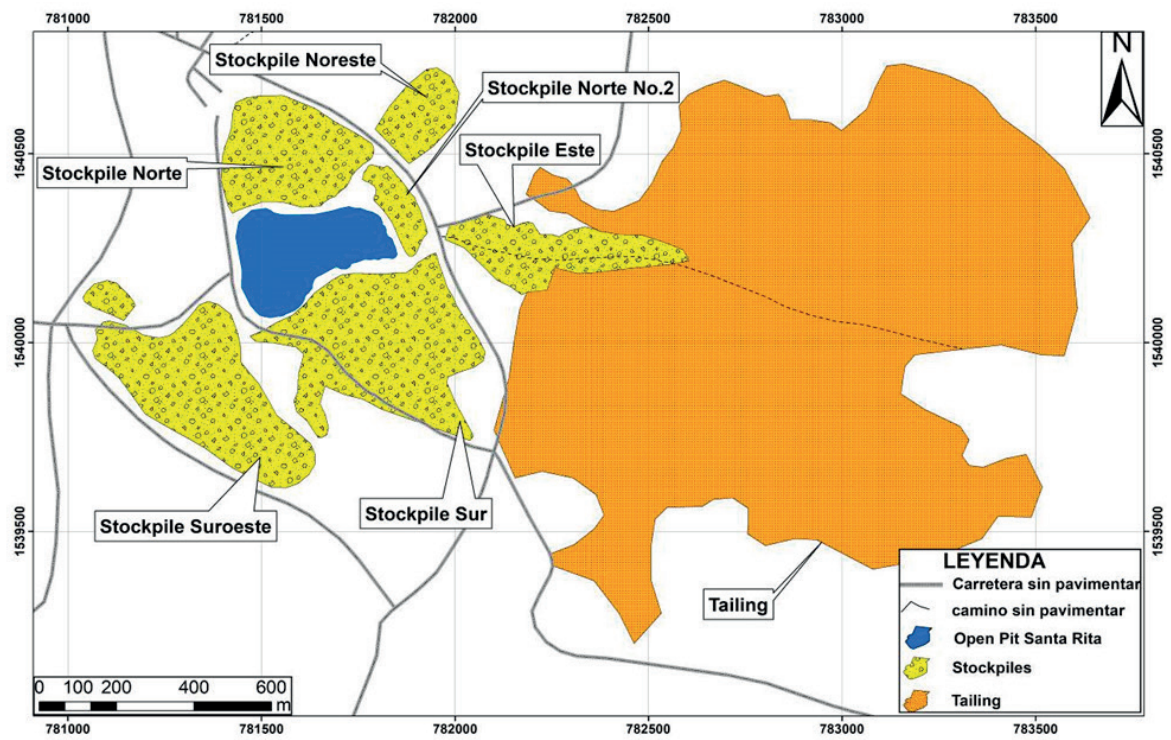

Figura 1. Ubicación de los stockpiles y tailing de Santa Rita. Fuente: Coffey Mining Pty Limited (2012). Coffey Mining Pty Limited was commissioned by Alder Resources Ltd. (the Optionee partner of Calibre Mining Corp.), to undertake a resource estimate in compliance with NI43-101 on the Rosita Copper-Gold-Silver Mine stockpiles in Nicaragua.

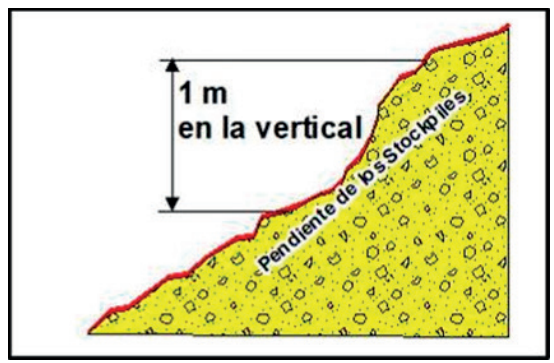

Figura 2. Muestras en canal stockpiles Santa Rita. Fuente: Salari \& Yungang Wu PGeo (2017). Rosita Mining Corporation NI 43-101 Preliminary Economic Assessment Study for the Santa Rita Project, Rosita, Nicaragua

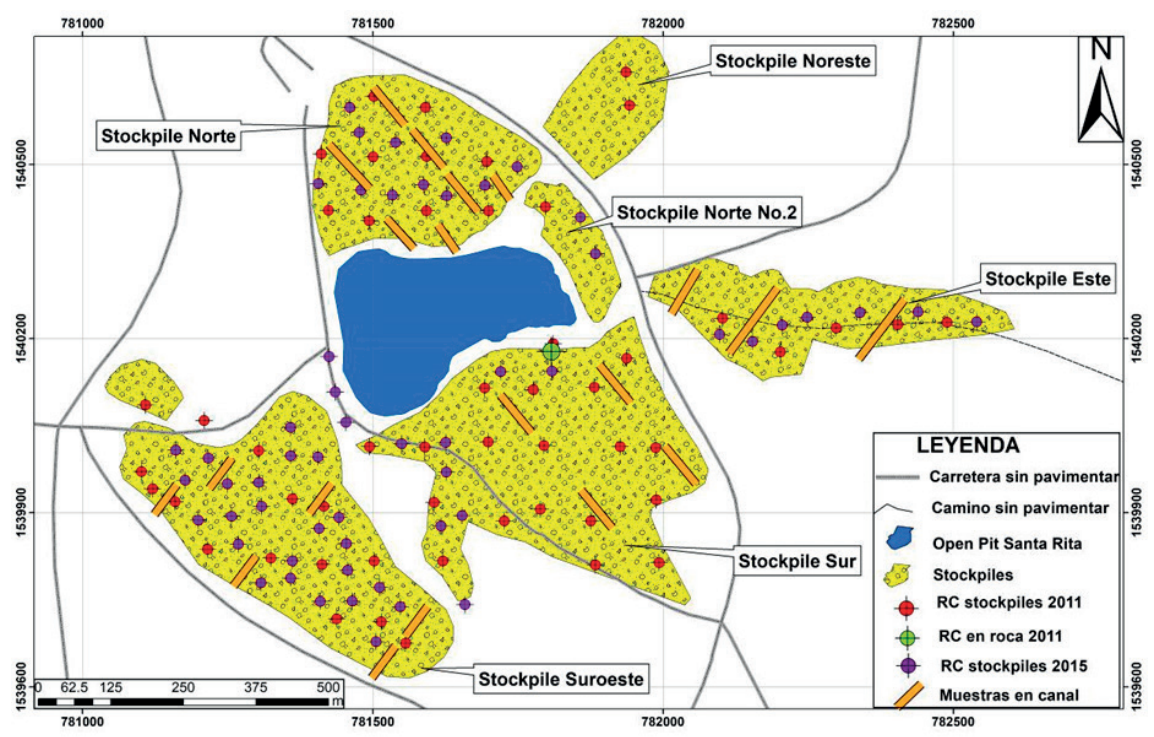

Figura 3. Localización de perforación tipo RC y Muestras en canal en los stockpiles Santa Rita. Fuente: Salari \& Yungang Wu PGeo (2017) Rosita Mining Corporation NI 43-101 Preliminary Economic Assessment Study for the Santa Rita Project, Rosita, Nicaragua 


\subsection{Exploración geológica detallada en los Tailing de Santa Rita}

En el área de los tailing se realizó una campaña de muestreo usando un barreno manual (auger por su nombre en ingles) en dos etapas:

1. La primera etapa se llevó a cabo en un área de aproximadamente $1.2 \mathrm{~km}^{2}$, la separación en cada sitio muestreado fue de aproximadamente $100 \mathrm{~m}$, la profundidad alcanzada fue entre $0.70 \mathrm{~m}$ a $3.70 \mathrm{~m}$

2. La segunda etapa de muestreo fue más detallada, y se usaron como referencia los resultados de la primera etapa, por lo tanto, el muestreo se realizó en un área de $0.16 \mathrm{~km}^{2}$. Cada sitio muestreado tiene una separación de $50 \mathrm{~m}$ y la longitud de cada muestra es de aproximadamente $2 \mathrm{~m}$. Ver Figura 4.

Con el objeto de evitar la contaminación y el colapso de los pozos durante la etapa de muestreo se introdujo un tubo tipo PVC con un diámetro de 3 pulgadas que funcionó como un "casing" (Ver Figura 5).

El auger se introdujo en el tubo pvc y se alcanzaron profundidades de hasta $7.20 \mathrm{~m}$. Todas las muestras tuvieron una longitud promedio de $2 \mathrm{~m}$ y atravesaron todo el espesor de los tailing.

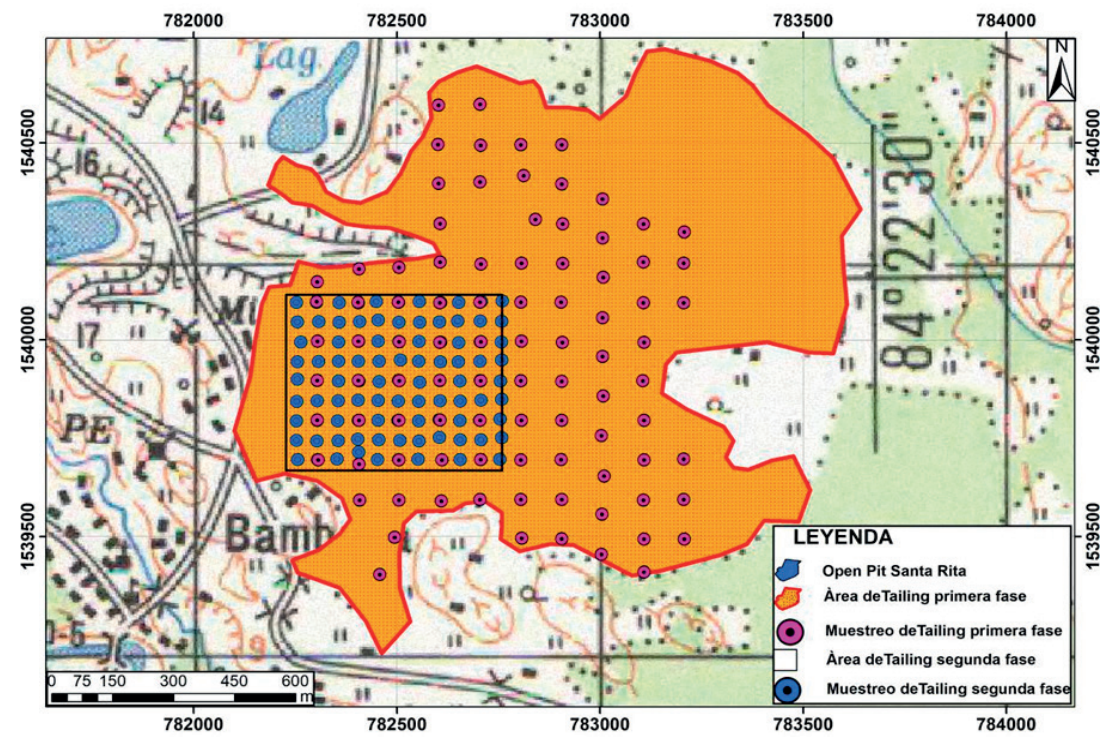

Figura 4. Ubicación de muestras Tailing primera y segunda fase. Fuente: Salari \& Yungang Wu PGeo (2017) Rosita Mining Corporation NI 43-101 Preliminary Economic Assessment Study for the Santa Rita Project, Rosita, Nicaragua ; (INETER, 1988).

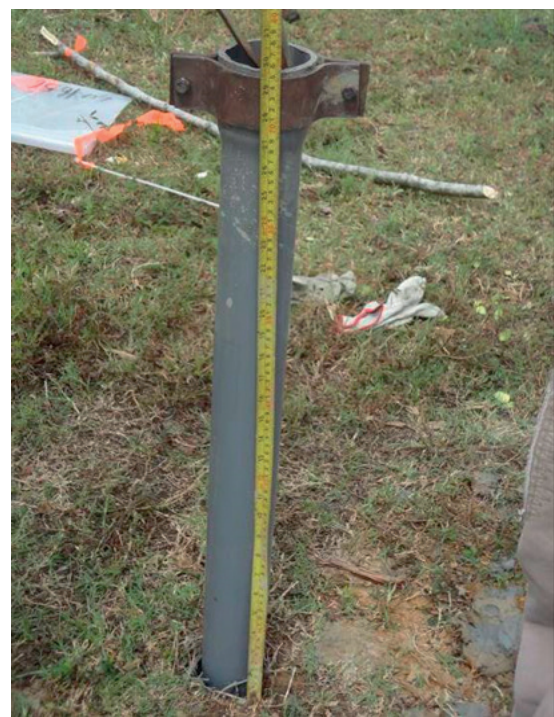

Figura 5. Tubo tipo PVC con un diámetro de 3 pulgadas que funcionó como un "casing en la toma de muestras de los tailings. Fuente: Elaboración propia. 


\section{RESULTADOS}

Los resultados obtenidos durante las etapas de exploración Geológica realizada en los stockpiles y tailing de Santa Rita se muestran a continuación.

\subsection{Resultados de la exploración geológica en los stockpiles de Santa Rita}

3.1.1 Resultados del muestreo en canal y dos etapas de perforaciones tipo Circulación Inversa (RC) (2011 y 2012) realizados en los stockpiles de Santa Rita.

De la toma de muestras en canales y las campañas de perforación tipo Circulación Inversa (RC) llevadas a cabo en (2011 y 2012) en los stockpiles, dieron resultados favorables y alentadores para continuar realizando más trabajos de exploración. Ver Tabla 1.

Coffey Mining Pty Limited was commissioned by Alder Resources Ltd. (the Optionee partner of Calibre Mining Corp.), to undertake a resource estimate in compliance with NI43-101 on the Rosita Copper-GoldSilver Mine stockpiles in Nicaragua

En la Tabla 1 se aprecian los primeros resultados de interés económico, demostrando el potencial y recursos existentes en los stockpiles, con valores promedio de $0.58 \%$ de $\mathrm{Cu}, 0.39 \mathrm{~g} / \mathrm{t} \mathrm{Au} \mathrm{y} 9.25 \mathrm{~g} / \mathrm{t} \mathrm{Ag}$.
3.1.2 Perforación tipo Circulación Inversa (RC) pozos

Infields cada 50 m de separación tercera etapa (2015). Los resultados obtenidos en esta tercera etapa de perforación (2015) permitió elevar la categoría de los recursos inferidos a indicados. Ver Tabla 2.

La Tabla 2 muestra la elevación de los recursos inferidos a indicados en los stockpiles y tailing de Santa Rita.

Rosita Mining Corporation NI 43-101 Preliminary Economic Assessment Study for the Santa Rita. Project, Rosita, Nicaragua

\subsection{Segunda fase de exploración en los stockpiles y tailing de Santa Rita}

3.1.2 Resultados del muestreo realizado en dos etapas en los tailing (2012 y 2013).

Gracias a los resultados obtenidos del primer muestreo realizado en los tailing en un área de $1.2 \mathrm{Km}^{2}$ permitió delimitar un área de menor tamaño $0.04 \mathrm{~km}^{2}$, pero con valores de mayor potencial, los cuales son considerados como recursos indicados.

Los mapas de anomalías geoquímica de $\mathrm{Cu}, \mathrm{Au}$ y $\mathrm{Ag}$, demuestran que el área de mayor interés se localiza

Tabla 1. Declaración de recursos existentes stockpiles de Santa Rita

\begin{tabular}{|c|c|c|c|c|c|c|c|c|c|}
\hline Stockpiles & Clase & $\begin{array}{c}\text { Tonelaje } \\
(1,000 t)\end{array}$ & $\begin{array}{l}\mathrm{Cu} \\
\%\end{array}$ & $\begin{array}{l}\mathrm{Au} \\
\mathrm{g} / \mathrm{t}\end{array}$ & $\begin{array}{l}\mathrm{Ag} \\
\mathrm{g} / \mathrm{t}\end{array}$ & $\begin{array}{c}\text { Cu Equi. } \\
\%\end{array}$ & $\begin{array}{c}\mathrm{Cu} \\
\text { Libras }\end{array}$ & $\begin{array}{c}\mathrm{Au} \\
\text { onzas }\end{array}$ & $\begin{array}{c}\mathrm{Ag} \\
\text { onzas }\end{array}$ \\
\hline Norte & Inferidos & 3.33 & 0.78 & 0.58 & 10.30 & 1.25 & 56.99 & 62,100 & $1,100,900$ \\
\hline Sur & Inferidos & 2.20 & 0.33 & 0.49 & 5.10 & 0.69 & 16.16 & 34,700 & 360,000 \\
\hline Noreste & Inferidos & 0.55 & 0.50 & 0.22 & 9.60 & 0.75 & 6.06 & 3,800 & 168,300 \\
\hline Este & Inferidos & 1.88 & 0.71 & 0.30 & 12.00 & 1.03 & 29.33 & 17,900 & 725,100 \\
\hline Total & Inferidos & 7.95 & 0.62 & 0.46 & 9.20 & 1.01 & 108.54 & 118,500 & $2,354,300$ \\
\hline
\end{tabular}

Fuente: Coffey Mining Pty Limited (2012)

Tabla 2. Recursos inferidos e indicados en los stockpiles de Santa Rita y tailing

\begin{tabular}{|c|c|c|c|c|c|c|c|c|}
\hline Stockpiles & Clase & $\begin{array}{c}\text { Tonne } \\
(1,000 t)\end{array}$ & $\begin{array}{l}\mathrm{Au} \\
(\mathrm{g} / \mathrm{t})\end{array}$ & $\begin{array}{c}\text { Contenido } \\
\text { Au } \\
(1,000 \mathrm{oz}) \\
\end{array}$ & $\begin{array}{l}\mathrm{Cu} \\
\%\end{array}$ & $\begin{array}{c}\text { Contenido } \\
\text { Cu } \\
(1,000 t) \\
\end{array}$ & $\begin{array}{l}\mathrm{Ag} \\
(\mathrm{g} / \mathrm{t})\end{array}$ & $\begin{array}{c}\text { Contenido } \\
\mathrm{Ag} \\
(1,000 \mathrm{oz})\end{array}$ \\
\hline \multirow{2}{*}{ Norte } & Indicados & 2,007 & 0.66 & 42.4 & 0.89 & 17.8 & 10.94 & 706.0 \\
\hline & Inferidos & 907 & 0.65 & 19.0 & 0.95 & 8.6 & 12.28 & 358.0 \\
\hline \multirow{2}{*}{ Este } & Indicados & 1,049 & 0.30 & 10.1 & 0.43 & 4.5 & 8.77 & 295.8 \\
\hline & Inferidos & 520 & 0.31 & 5.1 & 0.81 & 4.2 & 12.84 & 214.5 \\
\hline \multirow{2}{*}{ Sur } & Indicados & 800 & 0.52 & 13.5 & 0.46 & 3.7 & 5.88 & 151.1 \\
\hline & Inferidos & 634 & 0.43 & 8.9 & 0.29 & 1.9 & 3.90 & 79.5 \\
\hline \multirow{2}{*}{ Suroeste } & Indicados & 2,603 & 0.37 & 30.7 & 0.24 & 6.2 & 4.39 & 367.6 \\
\hline & Inferidos & 796 & 0.41 & 10.5 & 0.27 & 2.2 & 4.21 & 107.7 \\
\hline Noreste & Inferidos & 431 & 0.26 & 3.5 & 0.71 & 3.1 & 12.39 & 171.7 \\
\hline Norte No.2 & Inferidos & 150 & 0.68 & 3.3 & 0.71 & 1.1 & 5.42 & 26.1 \\
\hline \multirow{2}{*}{ Stockpile Total } & Indicados & 6,460 & 0.47 & 96.7 & 0.50 & 32.2 & 7.32 & $1,520.5$ \\
\hline & Inferidos & 3,437 & 0.46 & 50.3 & 0.61 & 21.0 & 8.66 & 957.5 \\
\hline Tailings & Inferidos & 1,956 & 0.56 & 35.2 & 0.21 & 4.0 & 9.65 & 607.0 \\
\hline
\end{tabular}

Fuente: Salari \& Yungang Wu PGeo (2017) 
en la parte inferior derecha del recuadro de los tailing (Ver Figuras 6, 7 y 8).

Es importante mencionar en este artículo que parte de los resultados también son los permisos ambientales requeridos para poder llevar a cabo todas las etapas de exploración en conjunto con los permisos de la comuna o alcadia municipal.

\section{DISCUSIÓN}

Los disturbios sociopolíticos en Nicaragua a finales de los años 70 s e inicio de los años 80 s junto con el bajo precio de los metales obligo abandonar y cerrar por completo la Mina Rosita.
El precio internacional de los metales es un factor determinante en la toma de decisiones para establecer un inicio o un fin de un proyecto minero, así como los factores modificadores.

La Mina Rosita dejó en los alrededores del tajo de Santa Rita volúmenes considerables de material de baja ley.

Los trabajos realizados a partir del año 2011 en los antiguos stockpiles y tailings de Santa Rita permitió cuantificar recursos, así como elevar la categoría de los mismos a inferidos e indicados.

Los recursos de la mina Rosita que antes eran considerados de baja ley y no tenían viabilidad económica en la actualidad mediante los trabajos de exploración se

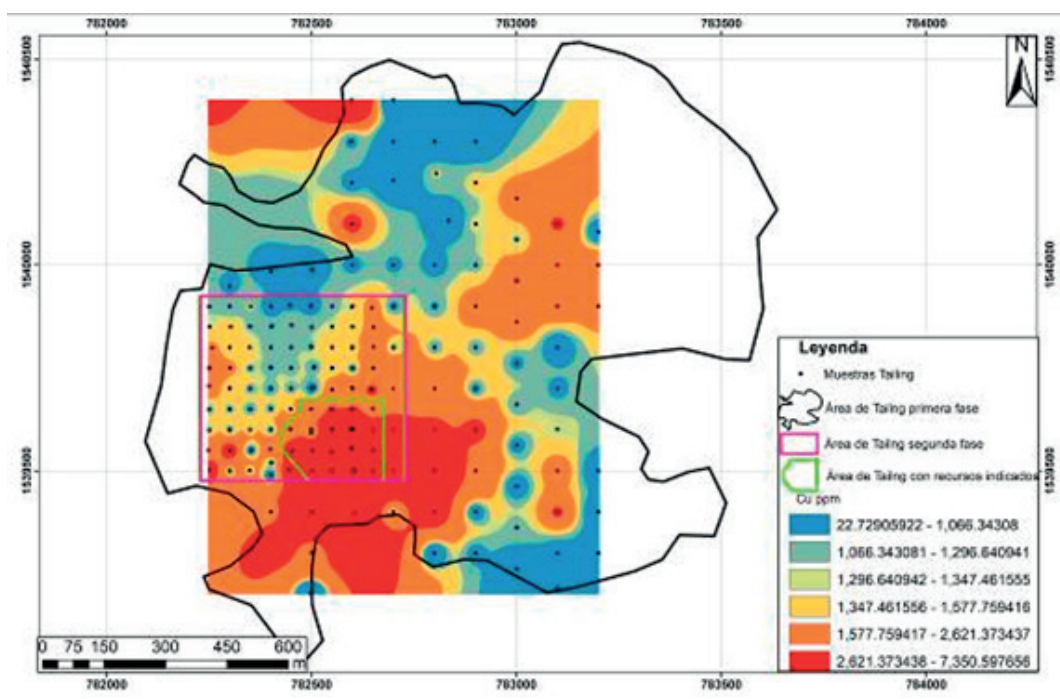

Figura 6. Ubicación de muestras y anomalías geoquímicas de Cu. Fuente: Salari \& Yungang Wu PGeo (2017) Rosita Mining Corporation NI 43-101 Preliminary Economic Assessment Study for the Santa Rita Project, Rosita, Nicaragua

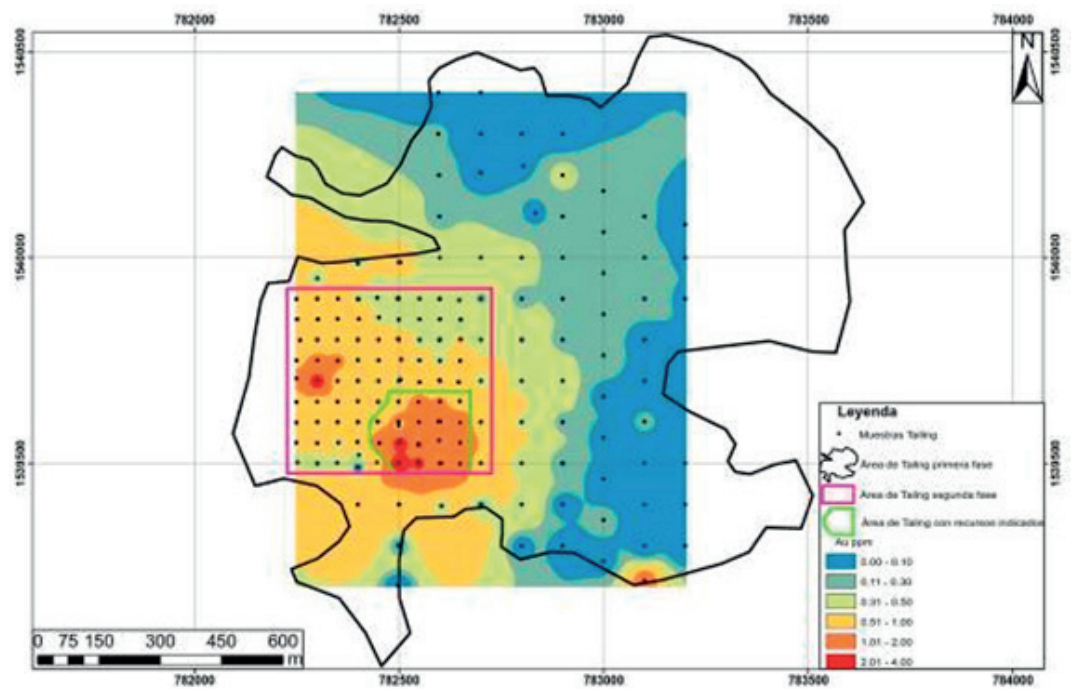

Figura 7. Ubicación de muestras Tailing y anomalías geoquímicas Au. Fuente: Salari \& Yungang Wu PGeo (2017) Rosita Mining Corporation NI 43-101 Preliminary Economic Assessment Study for the Santa Rita Project, Rosita, Nicaragua 


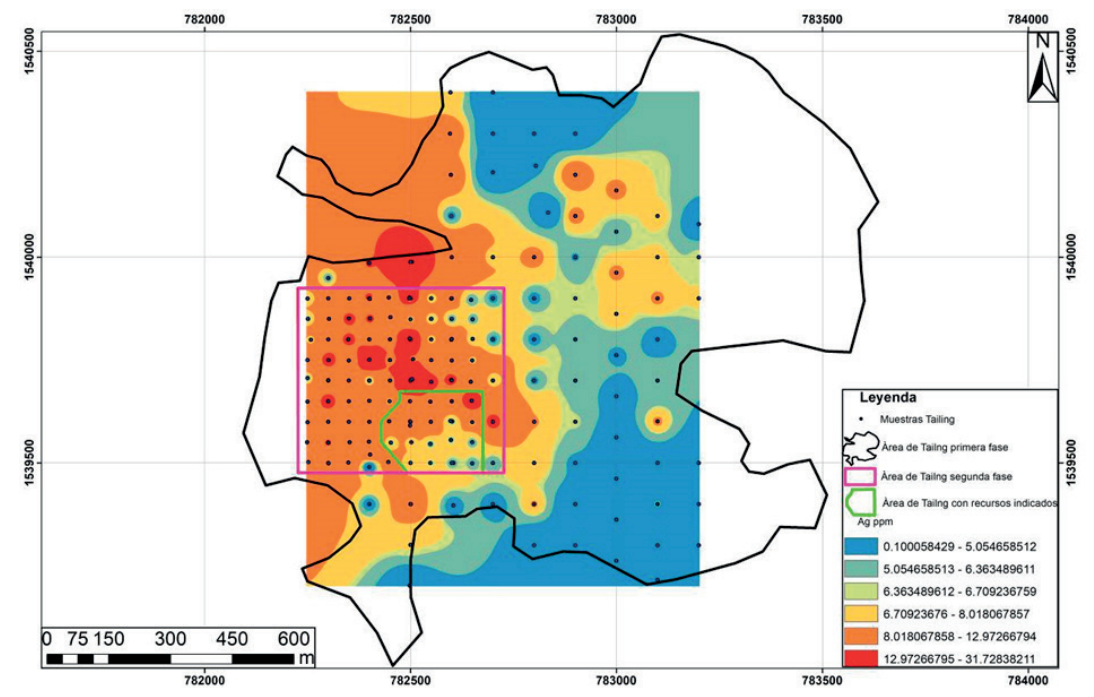

Figura 8. Ubicación de muestras Tailing y anomalías geoquímicas Ag. Fuente: Salari \& Yungang Wu PGeo (2017) Rosita Mining Corporation NI 43-101 Preliminary Economic Assessment Study for the Santa Rita Project, Rosita, Nicaragua

comprobó que su extracción es rentable dado su volumen, leyes minerales y bajos costos de procesamiento, así como el valor actual de los metales.

\section{CONCLUSIONES}

Con todos estos resultados finales los recursos antes existentes pasan a una categoría de recursos inferidos e indicado.

Los trabajos realizados en los stockpiles y tailing de Santa Rita demostraron la existencia de 125,840, 000 libras de $\mathrm{Cu}$ con ley mínima de $0.58 \%, 182,200$ onzas de $\mathrm{Au}$ con ley mínima de $0.4 \mathrm{~g} / \mathrm{t}$ de Au y 3,085,000 onzas de plata con ley mínima de $248 \mathrm{~g} / \mathrm{t}$.

El volumen de procesamiento para los stockpiles se estima en 328, 500 toneladas por año (1 a 3 años) y de 657,000 toneladas a partir del año 4.

En relación con los tailing el procesamiento se estima en 36,500 toneladas por año (1 a 3 años) y de 73,000 toneladas a partir del año 4 , con una estimación de vida de la planta de 10 años.

La ley mínima en los recursos inferidos e indicados, junto con los nuevos factores modificadores que antes no existían, permiten definir la viabilidad de la Mina Rosita, con VAN positivo, lo que hace atractivo este proyecto para los inversionistas

Lo antes mencionado se refleja en que se utilizó un precio del oro de US \$ 1200 / oz, un precio del cobre de US $\$ 2,5$ / lb y un precio de la plata de US \$16/ oz.

Los resultados de los recursos minerales presentados en esta publicación se estimaron utilizando el Canadian
Institute of Mining, Metallurgy and Petroleum, 2014.(CIM), Cim-Definition-Standards sobre Recursos y Reservas Minerales, Definiciones y Lineamientos preparados por la Comité Permanente de la CIM sobre Definiciones de Reservas y adoptado por el Consejo de la CIM (Canadian Institute of Mining, 2014).

\section{AGRADECIMIENTOS}

Un fraterno agradecimiento a la Unidad de Posgrado de la Universidad Nacional Mayor de San Marcos, por darme la oportunidad de presentar esta investigación, en especial al Ing. Sammy González por su dedicación y tiempo incondicional en todo momento.

\section{REFERENCIAS}

Canadian Institute of Mining, M. and P. (2014). Canadian Mineral Resource and Mineral Reserve Definitions. CIM MRMR Committee. https://mrmr.cim.org/en/standards/canadianmineral-resource-and-mineral-reserve-definitions/

Coffey Mining Pty Limited. (2012). NI 43-101 Technical Report on Mineral Resource Estimate of Rosita Stockpiles Calibre Mining Corp. https://calibremining.com/site/assets/ files/4006/rosita_mine_stockpile_report.pdf

INETER. (1988). Hoja Topográfica Mina Rosita 3357-4. https:// www.ineter.gob.ni/mapa/50k_1988/50K/3357-4.html

Salari, D. J., \& Yungang Wu PGeo, Pe. (2017). Preliminary Economic Assessment Study for the Santa Rita Project, Rosita, Nicaragua. www.denmengineering.com 